\title{
Parameters and indicators for providers of electronic publications evaluation
}

\author{
María Luisa Alvite Díez and Blanca Rodríguez Bravo \\ Área de Biblioteconomía y Documentación. \\ Facultad de Filosofía y Letras. Universidad de León \\ [University of Leon] \\ Leon, Spain \\ luisa.alvite@unileon.es \\ dphbrb@unileon.es
}

\section{Introduction}

Over the last few years, the first studies about suppliers of electronic publications have begun to appear. They have focused for the most part on evaluating the use of such publications by the academic community. Examples would be the recent work by Tenopir (2003) and, in Spain, that directed by Urbano (2004). Some of these studies, like those by Pullinger (1999) and by Eason, Richardson and Yu (2000), provide detailed information about the influence of contents, their coverage and relevance, and about the technical aspects that affect access and use.

The authors of this paper have carried out previous studies (2004) in which an individualized analysis was undertaken of two providers of electronic content ScienceDirect and Wiley InterScience-. Afterwards they lie $(2004,2005)$ in the area of comparative evaluation of information coverage and characteristics of interfaces, functions and services of five electronic content providers widely used by Spanish academic libraries: Emerald, Kluwer Online, ScienceDirect, SpringerLink and Wiley InterScience.

The study was undertaken within the framework of a research project financed by the Universidad de León ${ }^{1}$ for the years $2004 / 2005$. The chief objective of this research project has been to gain an overall knowledge of the provision of electronic information, its distribution and use by the academic community in order to allow universities and their libraries to negotiate with the large multinationals in the publishing sector on the basis of objective criteria on quality and utilization.

1. Evaluación de los proveedores de revistas electrónicas y estudio de la calidad de las publicaciones digitales (2004-2005). Investigador principal: Dra. Rodríguez Bravo. 


\section{Objectives and methodology}

The specific aims of this paper is to present an evaluation model and to share a methodology for the analysis comparative of information distributed, characteristics of interfaces and various functions and added value services of providers of electronic publications. This model has been applied to: Emerald, Kluwer Online, ScienceDirect, SpringerLink, and Wiley InterScience in order to test the validity of the methodology proposed. This analysis will permit the acquisition of trustworthy information on the nature and quality of these systems.

We reckon that works mentioned previously permitted testing the validity of the methodology proposed. Indicators are established to evaluate not just the extent of the materials provided, but also the access structure, characteristics of the interface, functions and added-value services.

The process of evaluation was organized around four parameters ( $\mathrm{A}-\mathrm{D}$ below), within which there were a number of grouped indicators as sub-parameters:

\section{A) Extension of contents}

- Horizontal Coverage: Volume of electronic publications.

- Thematic Coverage: Collections of scientific disciplines.

- Vertical Coverage: Retrospective reach of the contents.

The overall digital content available from the various suppliers was tallied up, including serial publications, electronic books, reference works, current protocols and databases. Browsing or navigational access by alphabetic order, categories and type of product routes, and via the listings given in information intended for librarians was used.

\section{B) Access Structure}

- Access Control Systems Used: Passwords, IP address authentication, digital certification and privileged users (superusers).

- Search and Navigation Performance: The levels and fields for searches, retrieval language, record of searches, browsing or navigation categories, fields with hypertext links, granularity of searches, and so forth.

- Presentation of Results: Descriptive data, criteria for ordering results and possibilities of ordering them, formats of publications, display of associated graphs and pictures, and similar.

A variety of queries were carried out so as to gain knowledge of the power of the retrieval tools, the range of simple and advanced search forms, the depth of the initial search, the relationships between the products distributed and the specific retrieval tools for some materials (reference works, current protocols and so on). 
The authors registered as users of the systems that required registration, in order to have access to capabilities restricted to registered users.

\section{C) Characteristics of the Interface}

- Design: Correct display, suitable layout for information, highlighting of elements, use of intuitively understandable forms and the like.

- Ergonomics: Characteristics and capacity for selection of items, speed and reliability of download and printing of entries, languages available and possibilities for personalizing the access page.

- User-friendliness: Syntax of messages, error messages, nature and suitability of user guides and help systems, possible system tips, and so forth.

The searches carried out were used to evaluate the characteristics of the interface and its suitability for users, particularly registered users. Error messages were induced and the help systems were reviewed.

\section{D) Functions and Added-value Services}

- Licensing Modes: Transparency of information and applicable law, contract availability for publications independently of subscription to the printed format, types of licence, guarantees offered to the licensee, rights to back issues, policies for digitizing, safety and privacy of data, multi-site use and access from outside the institution.

- Functions: User registers, quotation systems, keyword notification services, quotation alert functions and linking techniques used.

- Added-value Services: Teaching packages, new item sections, service to librarians, service to authors, integration with library services, standardization, statistical reports, and similar services.

A user profile was created to check requests for alerts and to check the Data Subscriber Interface. For the remaining points we have used the information presented in the licence held and offered by the firms on their central websites.

Exploration of the electronic packages was undertaken during the first half of 2004 and was revised during the month of September. For the evaluation, subscriptions taken out by the University of León were used, with the exception of Kluwer which was investigated through an open access route.

It should be note that in February 2005 was undertaken the integration of Kluwer's journals into SpringerLink package. Consequently, Kluwer platform doesn't exist longer. 


\section{Summary of results}

\section{A) Extension of contents}

The analysis includes serial publications, electronic books, reference works, current protocols and databases. Nevertheless, all analyzed providers take care of high-priority way of the electronic journals distribution.

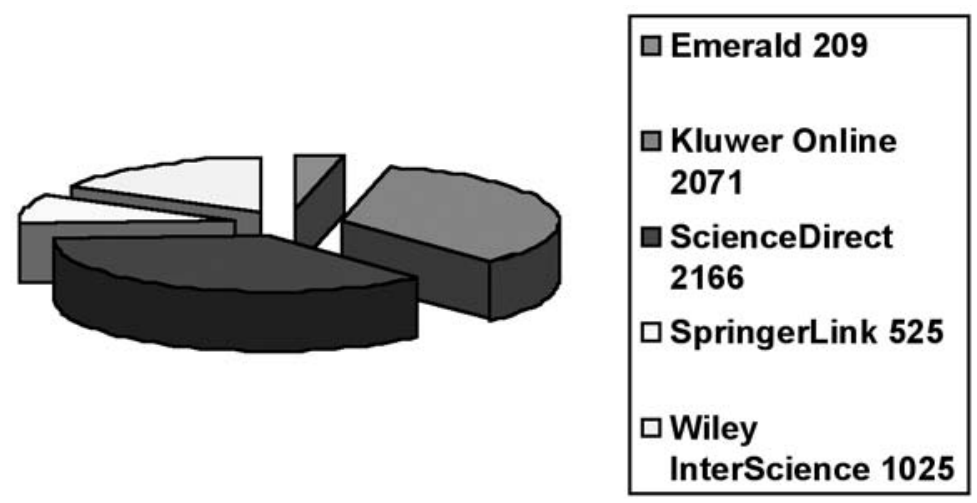

Kluwer Online and ScienceDirect stand out in contrast to the other providers because of their extensive offerings of electronic content. Wiley InterScience is a particularly good illustration of the three chief platforms for access to electronic materials on scientific and technical topics: digital journals, reference databases, and electronic books. Emerald concentrates on serial publications.

Springer's list is striking, as in its 30 collections of books there are no fewer than 2,537 individual publications. The number of electronic books available from Kluwer, at 1,210, is also relatively large. To a lesser degree, this is also true of ScienceDirect, whose 8 series of books run to 961 individual volumes, while Wiley InterScience has more than 500 on offer. Note should be taken of the section relating to reference works in the lists of Kluwer, Science and Wiley. The last of these three rounds out its materials with current protocols and reference databases. ScienceDirect similarly also distributes databases.

From their homepages Emerald, Science and Springer allow easy differentiation of contents available with the specific licence held, those items for which the institution has or does not have a subscription. Materials for which there is a subscription usually provide access to the full text, while non-subscribers only have access to reference details or summaries. As the University of León holds no licence from Kluwer Online, it wasn't possible to determine if this is true for that provider. It is also difficult to determine the full-text contents provided by Wiley InterScience when employing the browsing access routes offered to users from Wiley's web site. 
It should be noted that the language coverage for the material from all five providers is almost exclusively English, with a small amount of German and French.

The five providers of electronic contents studied are for the most part multidisciplinary. However, they basically offer publications from the areas Natural Sciences, Health Sciences and Social Sciences. In contrast, the presence of the Humanities in the packages analysed is limited, this being a field represented exclusively in the Kluwer and ScienceDirect lists.

If the area of Humanities is left out of account as having only a token presence in this supplier's range, the extent of thematic coverage offered by ScienceDirect certainly stands out, being noteworthy even in the area of Pure Sciences, which is the field having the smallest number of items. The primary orientation of this provider is towards Natural Sciences and Health Sciences.

Kluwer brings a considerable contribution to all of the thematic areas. The thematic range offered by Wiley InterScience is balanced, having almost a hundred publications even in the area in which it is most limited, Social Sciences. It is particularly strong in the field of Applied Sciences, fundamentally because of its large number of e-books in these disciplines. This provider lacks any Humanities materials.

Emerald is quite different, being almost exclusively concerned with the distribution of materials in the Social Sciences, mainly in Economics and Information Science. SpringerLink, on the other hand, has no involvement in the area of Social Sciences, being strongest fundamentally in Natural, Health and Pure Sciences. Nonetheless, this distributor does have a large number of single or monographic publications in the Applied Sciences in its series of books.

It is not easy to achieve an exact account of the start dates for the publications offered. This is because in some instances there are materials without dates and at other times there are discrepancies in the dates cited. This is made even more difficult by the coincidental complexity of the history of the periodicals involved: there are journals that have ceased publication; back issue files; journals that have changed names, been subdivided, incorporated or merged; and other complications.

The materials offered by ScienceDirect are noteworthy for their long chronological range, with some of its serial publications going back into the nineteenth century. Despite all this, Elsevier's incorporation en masse of journals really begins with the 1990s. A major increase of the availability of electronic materials during the 1990s is noticeable in all providers.

The currency of the collections for Wiley InterScience and to a lesser degree for ScienceDirect and Springer Link is evidenced by the large numbers of materials they hold that were published between 2000 and the time of our evaluation. 


\section{B) Access Structure}

In light of the results obtained for the indicators established, it is clear that all the suppliers perform adequately on the technical aspects that affect access to the content they offer.

A positive view was taken of systems that could recognize Internet Protocol numbers (IPs) for access. Passwords are required only if one desires to $\log$ on as a registered user, a feature that is not provided by Emerald. For their part, Emerald, Springer and Wiley all allow access via Athens login. For Wiley InterScience, there are superusers accredited for the ArticleSelect service.

The five providers evaluated permit searching of contents by indicating thematic categories for browsing or navigation. While the basic fields almost always have hypertext links, some suppliers use them more widely than others. In the case of Emerald, links extend to the classification and keywords, and Springer offers an interesting anchor or one-click point redirecting users to a range of possible links or "linking options".

It was seen as positive if systems had simple and advanced search forms, the latter so as offer a larger number of combinations and limit results by fields. On this point, the search forms for ScienceDirect stand out, being complemented with a Quick Search box.

Positive weighting was given to distributors with positional search boxes, adapted to the point that the user has reached, with an eye to limiting searches to within one publication, to the given volume, or the like. The systems considered here have individualized search engines for those cases in which there is provision of differentiated products: reference works, current protocols and even databases.

We considered it an advantage if there were possibilities for ordering results that the systems offer to users, with Wiley standing out through the use it makes of relevance, chronological order of appearance, and title, and with Kluwer adding the possibility of ordering by authors. All the products have both short-form and fullform presentation. The variant forms of information contained in the latter are exploited most effectively by Science and by Wiley.

The format most often used for full-text documents is portable document format (PDF). On formatting ScienceDirect stands out because it offers up to five different types of end-user presentation, and it also has a high percentage of documents in hypertext mark-up language (HTML). Individualized treatment of graphs and images, the quality of which can be crucial in some areas of academic endeavour, was positively valued.

In some cases there is room for improvement of features relating to access structures, primarily in relation to certain indicators under the sub-parameters for search and navigation capabilities and for presentation of results. While all providers allow 
searching on various fields and use basic operators, some do not permit recording of the history of searches and, with the exception of Emerald, there is no session history recorded.

\section{C) Characteristics of the Interface}

The evaluation of interfaces was in general good, from which it may be inferred that meticulous attention has been paid to their design and to an intuitively understandable user-system interaction.

In general, screens are acceptably readable, with appropriately contrasting colours, use of layout and indentation to facilitate reading, and suitable fonts and sizes for text. The organizational elements employed by all systems also seem appropriate, with icons or buttons that are sufficiently identifiable.

The use of check boxes and of drop-down menus in the forms offered to users was viewed positively, as was avoiding the direct use of range operators or qualifiers, which normally lead to greater complexity and can cause errors. We also recognize the indisputable usefulness of showing the search strategy employed and of highlighting in the results the specific terms used in the search. The second of these techniques is not in use by Emerald and Wiley.

The ergonomics sub-parameter took into account the facilities for customization available to the system administrator and the possibility of individual personalization of the interface, usually done through user profiles. The widest range here is offered by Kluwer, which allows personal choices for the output formats: typeface, colours, order of elements, and so on, all without need for individual registration.

With regard to the sub-parameter for user-friendliness all providers performed well relative to the indicator referring to the layout of information in differentiated blocks: formal description of the journal, authors, title, abstract, and so forth. A positive view was also taken of generalized and contextualized help support, which all the systems had to a greater or lesser degree. The user guides, tutorials and frequently asked questions of ScienceDirect stand out among these. However, the various systems' error messages are less well done and seldom are comments displayed to users to indicate possible typing errors or to suggest at least some change to fields when searches are unsuccessful.

\section{D) Functions and Added-value Services}

It is the final parameter, functions and added-value services, where the differences from one supplier to another are at their greatest. The provision of added-value services (course packs, news, and the like) is adequate, as are the variety of access routes (such as the availability of off-site use, pay-per-view modes, and so on). In contrast, the biggest deficiencies are to be seen in the functions sub-parameter, in 
those indicators relating to the quotation systems in use and to the possibility of requesting quotation alerts.

We consider it necessary for Spanish libraries to be able to have licence agreements drawn up in Spanish and covered by Spanish law when it comes to clearing up any disputes that might arise. Yet Kluwer and Springer have their contracts in English and the legislation applicable to their licences is German and Dutch respectively. For its part, Wiley makes its licences subject to American law.

A positive view was taken of a good range of types of licence and the possibility of access to materials in ways complementary to, or independent of, publication on paper, as is the case of E-Choice at ScienceDirect. It is worth pointing out that publishers like Wiley are currently considering distribution of their materials in a form suitable for personal digital assistant devices (PDAs). All the providers considered had options for pay-per-view. Among the models used for this, attention should be drawn to the novel vouchers or tokens for superusers from Wiley InterScience. If these are used on a publication up to an amount equivalent to $115 \%$ of its price, then it is automatically included by the supplier in the using establishment's licence.

In most cases, the guarantees offered to licensees can only be described as limited. Kluwer's commitment has led it to deposit its files at the Online Computer Library Centre (OCLC). The obligation accepted by the distributors to continue providing access to their collections of earlier issues is reasonable, and they have stated policies for back-number files. Guarantees on security and privacy of data are expressly stated by all of the firms.

In what they provide, suppliers have progressively included access routes complementary to what is available through an establishment's intranet, so that researchers can gain access to electronic materials from off-campus locations.

With respect to the sub-parameter relating to analysis of functions, it is necessary to point out the unusual case of Emerald, the sole provider not to make use of user registers. For the other suppliers of material, added-value services are largely tied to user registration. ScienceDirect is to be distinguished from the others, because its quotation system indicates articles that have cited the one being viewed and offers quotation alerts on this information. All the suppliers have notification or alert services. The linking techniques employed make use of the digital object identifier system (DOI) and CrossRef. Additionally, Wiley participates in CrossRef Search using Google technology.

The indicators making up the final sub-parameter show that all the suppliers perform adequately. All parts of the serial publications on offer are in electronic form, not just the academic articles in them. Production of teaching materials packs is facilitated and sections showing newly available items are offered. The provision 
of specific services for librarians and for authors is more uneven. Because of their added value, the Library Connect programme from ScienceDirect and the portals with information for thematic specializations at Emerald deserve particular notice.

ScienceDirect is particularly good with respect to the indicators for integration and standardization, presenting logos, search boxes and explicit facilities for linking with the online public access catalogue (OPAC) of the institution. It works with SGML/XML document type definitions (DTDs) for mark-up of documents and with other similar standards, such as MathML and XLink. Only ScienceDirect and Springer state that they participate in the COUNTER programme for gathering statistics.

\section{Discussion}

The evaluation of materials presented above makes it feasible to note the extensive general coverage, density per subject and long back runs of ScienceDirect. The analysis likewise points up the striking position of Kluwer, even more so in the area of electronic books. The horizontal coverage of information is similarly considerable in the Wiley InterScience package. These products, like Springer's, are encyclopaedic in nature, neglecting only the area of Humanities, present solely in Kluwer and, to a lesser degree, that of Science. By contrast, Emerald stands out because of its specialization and orientation towards social sciences.

The thematic organization for most of the suppliers considered could be improved. Only SpringerLink presents categories that are adapted to its contents under clear and self-explanatory labels. A smaller number of thematic classes for Emerald, Kluwer Online and ScienceDirect might be a better option, with subdivision into subcategories where necessary, the solution that Wiley InterScience successfully adopts.

It would be desirable for suppliers of packages to provide more trustworthy information about the contents covered: publications available, subjects to which they are assigned, what their retrospective reach is, whether they are complete texts or abstracts, formats available, and other points. It is difficult for licensees to check on these matters, which are critical matters for licensees in the contractual relationship being established.

The results obtained by ScienceDirect are to be noted, since, besides being the clear leader in the functions and added-value services area, ScienceDirect also brings together a powerful search engine, a flexible interface and a wide range of choices for display.

Wiley Interscience is noteworthy for holding the second place for the last three parameters. The good results for Kluwer's interface, and the correctness and balance of Emerald for the same parameters evaluated should likewise be mentioned.

Nevertheless, it seems appropriate to recommend the introduction of terminological tools to aid in carrying out conceptual searches, which are not satisfactorily 
covered simply by the use of keywords. The organization of results might in addition give priority to integrating solutions derived from similarity and clustering techniques.

Similarly, some aspects relating to standardization could be improved, as also the use of statistical reporting on the basis of the COUNTER model, essential for management of the collection by licensees.

It is necessary to carry out further studies complementary to the work reported here, so as to investigate the academic relevance of the electronic materials on offer, the overlap between providers and the facilities for access and functions provided by each of the systems. Such investigations into their quality, together with others covering the data on use of the products of electronic information suppliers, would be an essential empirical contribution to the international debate on the way academic periodicals will be acquired by the university libraries of the future and play a central role during the renegotiation of licences.

\section{References and Resources Consulted}

Alvite Díez, M. L.; Rodríguez Bravo, B. (2004). Análisis de la distribución de contenidos electrónicos de Wiley InterScience. El Profesional de la Información, Vol. 13, No. 3, pp. 209-220.

Alvite Díez, M. L.; Rodríguez Bravo, B. (2004). Análisis de la distribución de contenidos electrónicos de ScienceDirect. El Profesional de la Información, Vol. 13, No. 5, pp. 353-361.

Alvite Díez, M. L.; Rodríguez Bravo, B. (2005). Distribuidores de contenidos electrónicos: acceso, interfaz y funcionalidades. In 9as. Jornadas Españolas de Documentación. Madrid: Fesabid, pp 29-46.

Association of Research Libraries. (2000). Principles for Emerging Systems of Scholarly Publishing. [On line at: <http://www.arl.org/scomm/tempe.html.>] [Consulted on: 02-06-2004].

Bishop, A. P.; Buttenfield, B. P.; Van House, N. (eds.) (2003). Digital Library Use: Social Practice in Design and Evaluation. Cambridge, Massachusetts: The MIT Press. [Consulted.]

COUNTER (Counting Online Usage of NeTworked Electronic Resources). $<$ http://www.projectcounter.org/>.

Eason, K.; Richardson, S.; Yu, L. (2000). Patterns of Use of Electronic Journals. Journal of Documentation, Vol. 56, No. 5, pp. 477-504.

Jewell, T.D. (2001). Selection and Presentation of Commercially Available Electronic Resources: Issues and Practices. [On line at: $<$ http://www.clir.org/pubs/reports/pub99/pub99.pdf>]. Washington, D.C: Digital Library Federation. [Consulted on: 15-06-2004]. 
JISC Scholarly Communications Group. (2002). Final Report from the JISC Scholarly Communications Group (SCG) to the Research Support Libraries Group (RSLG). [On line at: $<$ http://www.jisc.ac.uk/uploaded_documents/rslg.pdf $>$ ]. [Consulted on: 21-05-2004].

Pullinger, D. (1999). Academics and the New Information Environment: The Impact of Local Factors on the Use of Electronic Journals. Journal of Information Science, Vol. 25, No. 2, pp. 164-172.

Rodríguez Bravo, B.; Alvite Díez, M. L. (2004). Análisis de los contenidos distribuidos por proveedores de publicaciones electrónicas. El Profesional de la Información, vol. 13, n. 6, pp. 441-449.

Rodríguez Bravo, B.; Alvite Díez, M. L. (2005). Survey of the Providers of Electronic Publications Holding Contracts with Spanish University Libraries. [On line at]. D-Lib Magazine, April, vol. 11, n. 4. <http://www.dlib.org/dlib/april05/alvite/04alvite.html>

Tenopir, C. (2003). Use and Users of Electronic Library Resources: An Overview and Analysis of Recent Research Studies. Washington, D.C.: Council on Library and Information Resources. [On line at: <http://www.clir.org/pubs/reports/pub120/pub120.pdf>].

Urbano, Cristóbal (ed.).(2004). Us de revistes electròniques de compra consorciada del CBUC (2000-2003). Informe realitzat per encàrrec del Consorci de Bibliotequees Universitàries de Catalunya. [On line at : <http://www.cbuc.es/5digital/Informe_revistes_CBUC_amb_grafics.pdf> ]

\begin{abstract}
Methodology for the analysis comparative of information distributed, characteristics of interfaces and various functions and added value services of electronic publications providers.
\end{abstract}

\title{
Keywords
}

Architecture of access, Electronic contents providers, Electronic publications, Evaluation, Functionalities, Information coverage, Interfaces, Methodology, Services of added value.

\section{Resumen}

Metodología para el análisis comparativo de los proveedores de publicaciones electrónicas: información distribuida, características de la interfaz y funciones y servicios de valor añadido. 


\section{Palabras clave}

Arquitectura de acceso, Cobertura de la información, Evaluación, Funcionalidades, Interfaces, Metodología, Proveedores de contenidos electrónicos, Publicaciones electrónicas, Servicios de valor añadido. 